\title{
Morphology and thermal properties of biodegradable poly(hydroxybutyrate-co- hydroxyvalerate)/tungsten disulphide inorganic nanotube nanocomposites
}

\author{
Tyler Silverman, Mohammed Naffakh, Carlos Marco, Gary Ellis
}

- Environmentally friendly INT $\mathrm{WS}_{2}$ is used to produce advanced PHBV NCPs

- Novel INT $\mathrm{WS}_{2}$ improve the thermal stability of PHBV

- INT $\mathrm{WS}_{2}$ is effective to accelerate the crystallization of PHBV

- Ring banded spherulites of PHBV are observed at low INT $\mathrm{WS}_{2}$ contents.

- The benefits of using INTs compared to other nanoscale fillers are highlighted.

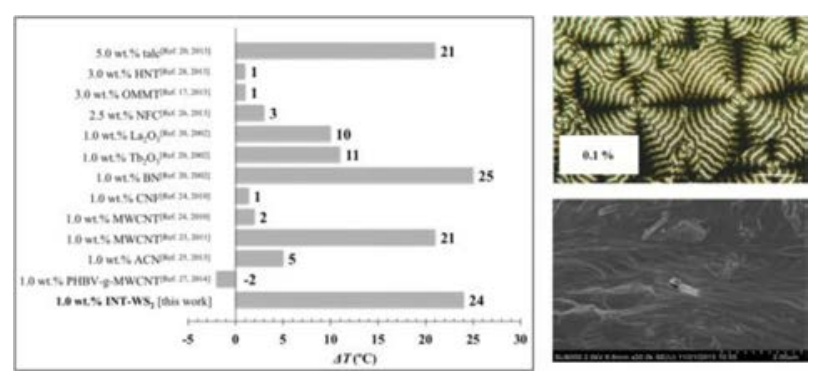

a b s t r a c t

Promising biodegradable and renewable poly(hydroxybutyrate co hydroxyvalerate) (PHBV) nano composites based on tungsten disulphide inorganic nanotubes (INT $\mathrm{WS}_{2}$ ) were efficiently prepared by a simple solution blending method The structure, morphology, thermal stability and crystallization behavior of the nanocomposites were investigated by ultra-high field emission scanning electron mi croscope (FESEM), thermogravimetric analysis (TGA), differential scanning calorimetry (DSC), wide angle $\mathrm{X}$ ray scattering (WAXS) and polarized optical microscopy (POM) techniques. As previously observed in poly(3 hydroxybutyrate) (PHB) hybrid systems, the dispersion, morphology and thermal properties of PHBV/INT $\mathrm{WS}_{2}$ nanocomposites could be tuned by the introduction of small amounts of INT $\mathrm{WS}_{2}$. The results revealed that a good dispersion of INT $\mathrm{WS}_{2}$ in the PHBV matrix influenced the morphology and non-isothermal crystallization behavior of PHBV that depends on both the INT $\mathrm{WS}_{2}$ concentration and the cooling rate. A significant enhancement in thermal stability of PHBV and a highly efficient nucleating effect of the INT $\mathrm{WS}_{2}$ comparable to specific nucleating agents or other nano sized fillers was observed. These observations are of importance for extending the practical applications of these biopolymer nanocomposites towards eco-friendly (e g sustainable packaging) and biomedical (e g bone tissue engineering) applications

1. Introduction

In 2015 it is estimated that around 300 million tons of plastic materials will be produced globally, mainly from petroleum 
feedstocks, and as a result there is growing concern about their future environmental and fiscal impact [1]. In the meantime the uncontrollable economic fluctuations in crude oil clearly indicate that supplies are finite, and "white pollution" originating from mainstream plastics creates an acute problem around the world. Thus, the challenge for scientists and engineers, both in academic and industrial frameworks, to develop alternative bio based and biodegradable plastic materials is becoming a critical issue with important objectives that must have projected impact within a decade.

In order to address the aforementioned problems, considerable attention has been given in the last two decades to poly hydroxyalkanoate (PHA) biopolyesters, a family of renewable and biodegradable thermoplastics. The production of PHA biopolymers originates in genetically controlled, energy storage procedures of a number of microorganisms under certain environmental situations $[2,3]$. Poly(hydroxybutyrate co hydroxyvalerate) (PHBV), the most common PHA copolyester, can be transformed using conventional thermoplastics processing equipment, and has properties compa rable to a range of petroleum based plastics, making it appealing as a biodegradable engineering material. It is highly anticipated as a potential substitute for applications with ephemeral functionality (packaging, pharmacology, farming, etc.) as well as a possible contender for advanced, non toxic biomedical applications (time released drug delivery vehicles, biodegradable tissue restoration scaffolds, etc.) [2-6]. However, PHBV typically has a high inherent degree of crystallinity ( $\geq 50 \%$ ), and despite being biodegradable and of biological origin, it has not achieved major commercial pros perity. Like other bio based materials, there are economic, ener getic, and mechanical drawbacks for their industrial success, and in particular for PHBV, it has slow crystallization kinetics and a ther mal stability limit that is close to the melting point $\left(\sim 170-180^{\circ} \mathrm{C}\right)$ of the polymer [7-9]. During the extrusion process, for example, the copolyester would experience local temperatures higher than that of the bulk due to high shear friction from the rotating screws, leading inevitably to the pre mature onset of degradation of the material. In molding applications, long mold injection cycles are required due to slow crystallization, which would cause low pro ductivity and expend large amounts of energy. Likewise, a spun film of PHBV would be more prone to self adhesion because it remains sticky due to slow conversion from the amorphous phase to the crystalline phase even after cooling.

Thus, in order to compete with commodity plastics, new stra tegies to improve mechanical, thermal, rheological, and economic limitations of PHBV as an eco friendly biomaterial are of current interest. One approach has involved blending PHBV with other polymers such as poly(L lactic acid), poly(dicyclohexylitaconate), poly(ethylene oxide), poly(ethylene succinate), poly( hydroxybutyrate), poly(butylene adipate co terephthalate), or poly(propylene carbonate) [10-16]. Other improvement strategies include incorporation of plasticizers such as acetyl tributyl citrate (ATBC) [17] or modified processing techniques such as post annealing [9]. Also, the incorporation to PHBHV of certain addi tives to create composite, binary or higher, materials that act as heterogeneous nucleating agents (NA) to enhance the crystalliza tion kinetics has been extensively studied, and include kenaf fibers, talc, boron nitride $(\mathrm{BN})$, lanthanum oxide $\left(\mathrm{La}_{2} \mathrm{O}_{3}\right)$, terbium oxide $\left(\mathrm{Tb}_{2} \mathrm{O}_{3}\right)$, wood fiber, cyanuric acid (CA), and raw coir [18-22]. More recently new approaches have appeared using low concentrations of nanoscale particles (i.e. nanoparticles) to create nanocomposite systems in order to improve physical and thermal properties of plastics. The known nanocomposite systems for crystallization studies of PHBV based materials include small weight fractions of organo modified montmorillonite (OMMT), carbon nanotubes (CNT), carbon nanofibers (CNF), acetylated chitin nanocrystals
(ACN), nanofibrillated cellulose (NFC), multi walled CNT grafted with PHBV (PHBV g MWCNT), and halloysite nanotubes (HNT) [17,23-28]. Nanoparticles have huge surface area to volume ratios and are incorporated with a view to creating extensive polymer -nanofiller interactions with the potential to yield interesting mechanical and thermal properties. However, most nanoparticles have high surface energies and, as a result, they typically form agglomerates unless chemically modified. Poorly dispersed nano structures impedes nanocomposite formation and consequently diminishes any potential property improvements. Sanchez Garcia et al. reported that carbon nanotubes had a limited effect on increasing overall crystallization rates in PHBV [24], attributed to poor nanoparticle distribution, whilst Shan et al. used surface modified CNT and reported excellent increases in crystallization rates of PHBV [23].

Inorganic nanotubes of tungsten disulfide (INT $\mathrm{WS}_{2}$ ) were first reported in the early 1990s by Tenne et al. [29]. In the following years, the development of many other inorganic nanotubes (INT) followed and, due to their unique electronic configurations, pro cessing techniques, and other chemomechanical properties, these are expected to create superb nanocomposite materials [29-38]. Unmodified INT $\mathrm{WS}_{2}$ are low cost, environmentally friendly and biocompatible nanofillers since they possess much lower cytotox icity than other nanoparticles, such as, silica or carbon black [39]. In particular, these novel nanomaterials have demonstrated inter esting properties such as high impact resistance, flexibility under tensile stress, superior tribological behavior, and fracture resistance from shockwaves [38]. With such excellent properties, and rela tively simple and inexpensive fabrication, the incorporation of INT $\mathrm{WS}_{2}$ in polymer systems has become a field of recent interest as it opens opportunities for many new applications [40-46]. The pre sent research continues work in this field and focuses for the first time on the use of INT WS $\mathrm{W}_{2}$ as a novel nanoreinforcement for enhancing the processability and performance of PHBV. The influ ence of the INT concentration on morphology and thermal prop erties is analyzed in detail. In particular, calorimetric studies were undertaken in order to determine the kinetic parameters during non isothermal (dynamic) crystallization from the melt, since these conditions are closest to industrial processing conditions.

\section{Experimental section}

\subsection{Materials and processing}

PHBV biopolymer with 2.0 wt.\% hydroxyvalerate (HV) content was purchased from GoodFellow Cambridge, Ltd in powder form $\left(\mathrm{M}_{\mathrm{w}} \quad 410 \mathrm{~kg} \mathrm{~mol}^{-1}\right)$ and used as received. The tungsten disulfide inorganic nanotubes (INT $\mathrm{WS}_{2}$ ) were obtained from NanoMaterials, Ltd (Israel) and used without chemical modification. Various for mulations of PHBV/INT $\mathrm{WS}_{2}$ nanocomposites were prepared by dispersing INT WS 2 (0.1, 0.5 and 1 wt.\%) in a solution of PHBV in chloroform, then precipitated in methanol and subsequently filtered and dried in a vacuum oven at $50{ }^{\circ} \mathrm{C}$ for $24 \mathrm{~h}$. Then, the samples were pressed into films of $0.5 \mathrm{~mm}$ thickness in a hot press system at $180{ }^{\circ} \mathrm{C}$ using two heating/cooling plates.

\subsection{Characterization techniques}

Surface images using an ultra high field emission scanning electron microscope (FESEM) SU8000 (Hitachi Co., Japan) were obtained from samples fractured in liquid nitrogen and coated with a $\sim 5 \mathrm{~nm}$ chromium.

The thermal stability of the control and binary systems were recorded from room temperature to $600{ }^{\circ} \mathrm{C}$ at a heating rate of $10{ }^{\circ} \mathrm{C} \mathrm{min}^{-1}$ by thermogravimetric analysis (TGA) using a TA 
Instruments Q50 thermobalance in an oxidative (air) atmosphere.

Non isothermal (dynamic) crystallization of the neat PHBV and PHBV/INT WS ${ }_{2}$ nanocomposites was measured using a Perkin Elmer DSC7/Pyris differential scanning calorimeter (DSC) (Perkin Elmer Espana SL, Madrid, Spain), calibrated with indium

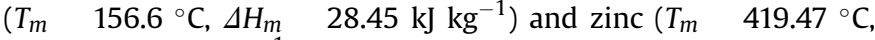
$\Delta H_{m} \quad 108.37 \mathrm{~kJ} \mathrm{~kg}^{-1}$ ). All samples of between 6 and $11 \mathrm{mg}$ weight were placed in sealed $40 \mu \mathrm{L}$ aluminum pans in a nitrogen flowing atmosphere. The thermal cycle employed consisted in holding the samples at $180^{\circ} \mathrm{C}$ for 5 min to erase any thermal histories [20-22] and subsequently cooling to $40^{\circ} \mathrm{C}$ at six different cooling rates $(\phi)$ : $2,5,10,15,20$ and $25^{\circ} \mathrm{C} \mathrm{min}{ }^{-1}$. After the dynamic crystallization process, the samples were heated at a rate of $5{ }^{\circ} \mathrm{C} \mathrm{min}^{-1}$ to the aforementioned holding temperature. The crystallization temper ature $\left(T_{p}\right)$ were determined from the minimum of the crystalliza tion exotherm observed during the cooling scan, respectively. The

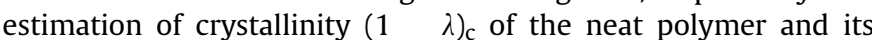
nanocomposites was calculated by:

$$
(1 \quad \lambda)_{c} \frac{\Delta H_{c}}{\Delta H_{m}^{0}}
$$

where $\Delta H_{c}$ is the apparent crystallization enthalpy and $\Delta H_{m}^{o}$ is the enthalpy of melting for perfect crystals: $146 \mathrm{~J} \mathrm{~g}^{-1}$, which is appro priate for this copolymer of low HV content $[2,17]$.

Wide angle $X$ ray scattering (WAXS) experiments of pure PHBV and PHBV/INT WS $\mathrm{W}_{2}$ nanocomposites (0.1 wt.\% sample excluded from WAXS) were performed using a Bruker D8 Advance diffrac tometer (Bruker AXS GmbH, Karlsruhe, Germany) with Ni filtered $\mathrm{Cu} \mathrm{K} \alpha$ radiation ( $\lambda \quad 1.5418 \AA$ ) over a double Bragg angle $(2 \theta)$ regime between $12^{\circ}$ and $40^{\circ}$ with a resolution of 42 points per degree. The equipment includes a temperature controlled chamber to analyze the dynamic crystallization and melting behavior over a broad range of temperatures. Each sample was held at $180{ }^{\circ} \mathrm{C}$ for $5 \mathrm{~min}$, then cooled to $30^{\circ} \mathrm{C}$ and subsequently heated to $180{ }^{\circ} \mathrm{C}$, both at $\phi \approx 7{ }^{\circ} \mathrm{C} \min ^{-1}$.

The crystalline morphology of the samples was examined with a Reichert Zetopan Pol polarized light microscope (POM). Samples were melted and compressed between two glass slides at $180^{\circ} \mathrm{C}$ for 5 min in a Mettler FP82HT hot stage. All samples were cooled to room temperature $\phi \quad 2$ and $10^{\circ} \mathrm{C} \mathrm{min}^{-1}$ and single images were obtained at room temperature.

\section{Results and discussion}

\subsection{Dispersion of INT $W S_{2}$ in the PHBV matrix}

The dispersion of the nanoparticles in the polymer matrix as well as interfacial interaction between the polymer matrix and nanoparticles play important roles in the crystallization behavior of polymers. Fig. 1a and b show an overview of the fracture surfaces of the PHBV/INT WS $\mathrm{W}_{2}$ nanocomposites with 0.5 and $1.0 \mathrm{wt} . \%$ of INT $\mathrm{WS}_{2}$, respectively. It can be clearly seen that INT $\mathrm{WS}_{2}$ are well dispersed within the PHBV matrix without any apparent aggrega tion. It is important to point out that the INT $\mathrm{WS}_{2}$ do not require exfoliation or chemical modification, making it possible to obtain new biopolymer nanocomposite formulations without the complexity and processing costs associated with such treatments. In particular, the efficient dispersion of INT $\mathrm{WS}_{2}$ at the nanoscale is likely related to the lubricant character of these inorganic nano tubes and the consequent improvement in tribological properties $[45,46]$. Thus, the INT $\mathrm{WS}_{2}$ acted as lubricant nanoadditives during the processing of the PHBV biopolymer nanocomposites. An anal ogous behavior has been reported for PHB and PEEK based com posites reinforced with INT $\mathrm{WS}_{2}[45,46]$.
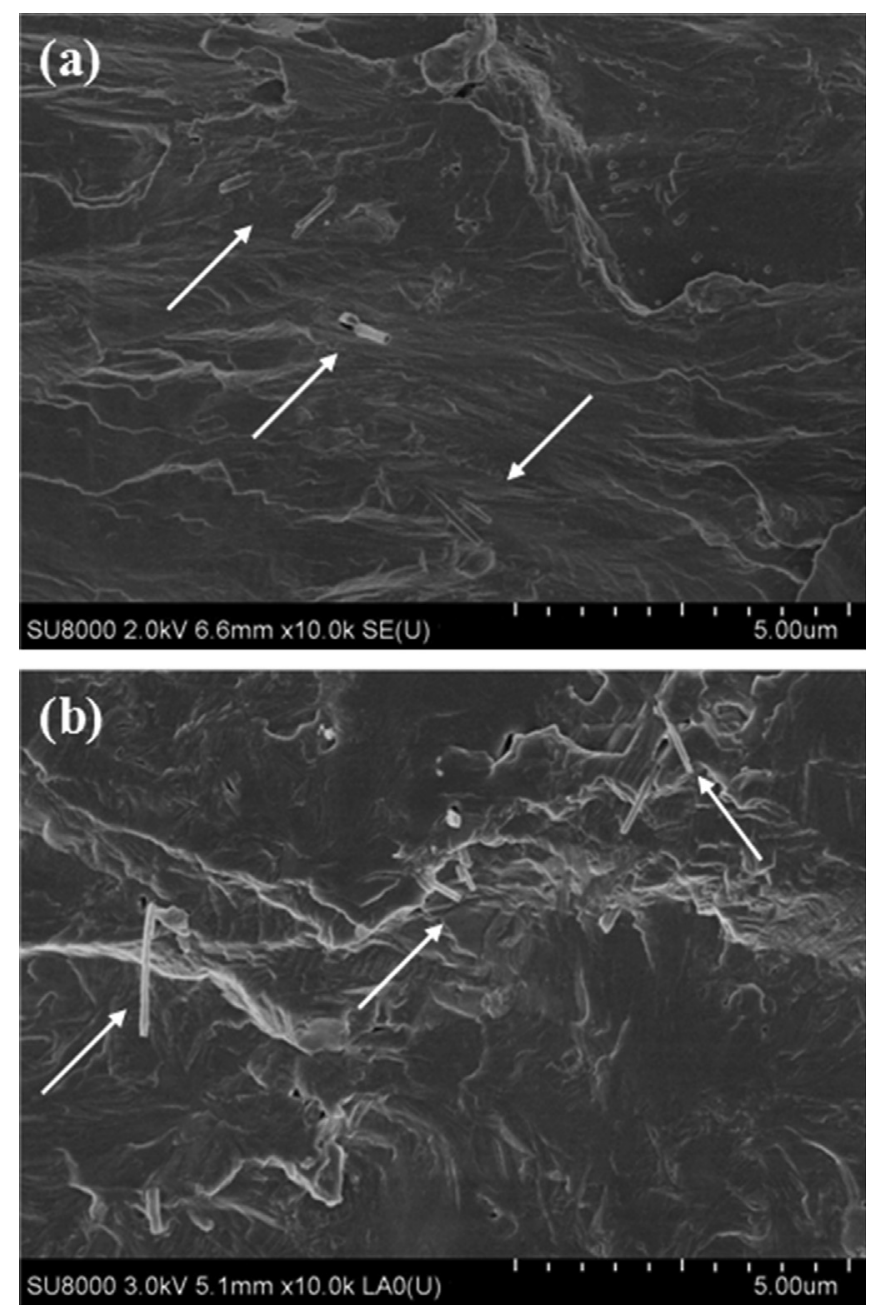

Fig. 1. FESEM image of PHBV/INT-WS ${ }_{2}$ nanocomposites. (a) PHBV/INT-WS 2 (0.5 wt.\%) and (b) PHBV/INT-WS 2 (1.0 wt.\%).

\subsection{Thermal stability of PHBV nanocomposites with INT $W S_{2}$}

Thermogravimetric analysis (TGA) of the PHBV/INT $\mathrm{WS}_{2}$ nano composites in air was studied to determine the effect of the inor ganic nanotube concentration in the PHBV matrix. TGA studies help establish limiting processing conditions for thermoplastic mate rials, which is very important for PHBV with its narrow processing window. An initial degradation temperature $\left(T_{i}\right)$ was marked when the original sample weight decreased by $2 \%\left(T_{2} \%\right)$ during the heating process. For neat PHBV and the 0.1 wt.\% INT WS 2 nanocomposite there was no noticeable difference, whereas for the $0.5 \mathrm{wt} . \%$ and 1.0 wt.\% nanocomposites $T_{i}$ increased by almost $10^{\circ} \mathrm{C}$ and $20^{\circ} \mathrm{C}$, respectively, as shown in Fig. 2, which is very important for PHBV with its narrow processing window. The temperature at which thermal degradation achieves its maximum rate $\left(T^{\prime}\right)$ also occurred at higher temperatures for the 0.5 and $1.0 \mathrm{wt} . \%$ nanocomposites. However, this trend was not observed for the reported poly(3 hydroxybutyrate) (PHB)/INT WS $\mathrm{W}_{2}$ nanocomposites by varying the INT $\mathrm{WS}_{2}$ concentration [44]. The addition of INT $\mathrm{WS}_{2}$ had no in fluence on the thermal stability of PHB matrix. Therefore, the noticeable degradation temperature increment observed in the PHBV copolyester could be attributed to a remarkable stabilization effect induced by the strong adhesion and homogenous dispersion of the INTs within the matrix, combined with the steric effect of HV units in the PHBV/INT WS 2 systems. Consequently, the presence of 


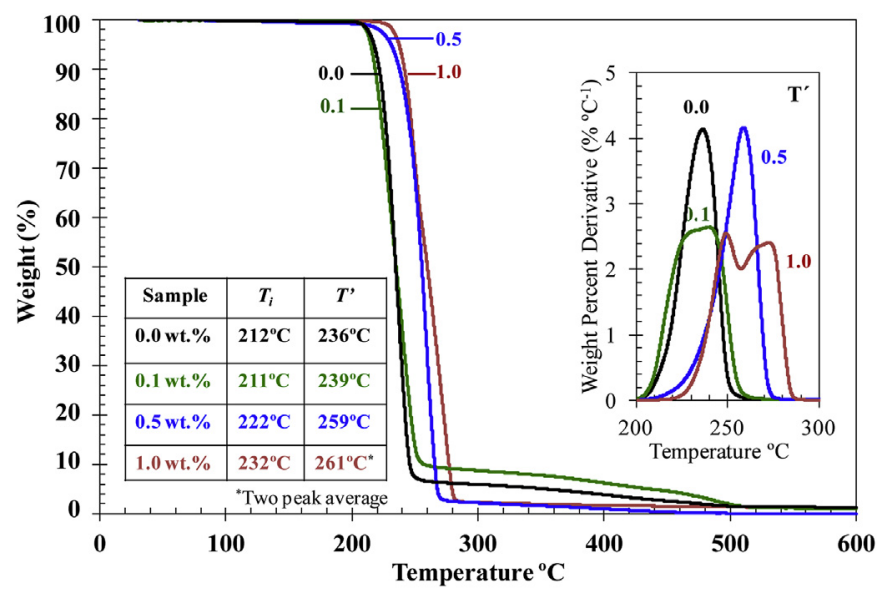

Fig. 2. TGA curves of PHBV and PHBV/INT-WS 2 nanocomposite systems in air.

INT WS $\mathrm{W}_{2}$ and HV effectively hinders the diffusion of degradation products from the bulk of the polymer to the gas phase, hence slowing down the decomposition process.

\subsection{Non isothermal crystallization behavior}

In semicrystalline polymers, the crystallization behavior gov erns not only the crystalline structure and morphology, but also the final mechanical properties of the material. PHBV is a semi crystalline polymer that can crystallize from either molten or glassy states, corresponding to melt crystallization and cold crystallization, respectively. The widespread industrial use of PHBV as a biodegradable material is mostly limited by its slow crystalline transformation. Therefore, if poly(hydroxybutyrate co hydroxyvalerate) based plastics are to be exploited in the market place as eco friendly and biologically apt materials, a comprehen sive understanding of the kinetics of non isothermal (dynamic) crystallization, i.e. crystallization rate, crystal structure, nucleation activity, effective energy barrier, etc., is of significant importance.

As underlined in the experimental section, an unfilled PHBV control sample and INT filled nanocomposites with three different compositions were tested under six nonisothermal conditions from $180^{\circ} \mathrm{C}$ to $40^{\circ} \mathrm{C}$ at $\phi$ ranging from 2 to $25^{\circ} \mathrm{C} \mathrm{min}^{-1}$, corresponding to temperature variations commonly found in thermoplastic fabrica tion setups. For all samples, the slower cooling rates showed higher crystallization peak temperatures $\left(T_{p}\right)$ and inversely, faster cooling rates showed lower $T_{p}$. For example, cooling from the melt at $\phi \quad 25{ }^{\circ} \mathrm{C} \min ^{-1}, \mathrm{~T}_{\mathrm{p}, \text { PHBV }} \quad 76.5^{\circ} \mathrm{C}$ and, at $\phi \quad 2{ }^{\circ} \mathrm{C} \min ^{-1}, \mathrm{~T}_{\mathrm{p}}$, PHBV $102.4^{\circ} \mathrm{C}$. The DSC cooling curves corresponding to these and other $\phi$ for unfilled PHBV are shown in Fig. 3a. As expected, at slower $\phi$ more time was available for polymer chain reorganization, the initiation of homogeneous nucleation, and subsequent chain growth into crystalline domains. On the contrary, at faster $\phi$ from the molten state macromolecular chain mobility was rapidly impeded with unsufficient time for the polymer chains to organize into crystalline domains, thereby shifting the entire crystallization process to lower temperatures, as observed in the DSC cooling curves. Fig. $3 b-d$ show the DSC cooling curves obtained from PHBV/INT WS 2 nanocomposite systems at the same $\phi$ tested in the neat PHBV. It can be observed that the addition of as little as 0.1 wt.\% INT $\mathrm{WS}_{2}$ generates a significant shift to higher tempera tures of $T_{p}$ for all cooling rates, Fig. $3 \mathrm{~b}$. At higher INT WS 2 concen trations the values of $T_{p}$ continued to increase, tending to plateau at the highest concentration of 1.0 wt.\%. For example, at $\phi \quad 10{ }^{\circ} \mathrm{C} \min ^{-1}, \mathrm{~T}_{\mathrm{p}, \text { PHBV }} \quad 88.0{ }^{\circ} \mathrm{C}, \mathrm{T}_{\mathrm{p}, 0.1 \mathrm{wt} . \%} \quad 100.4{ }^{\circ} \mathrm{C}, \mathrm{T}_{\mathrm{p}}$,
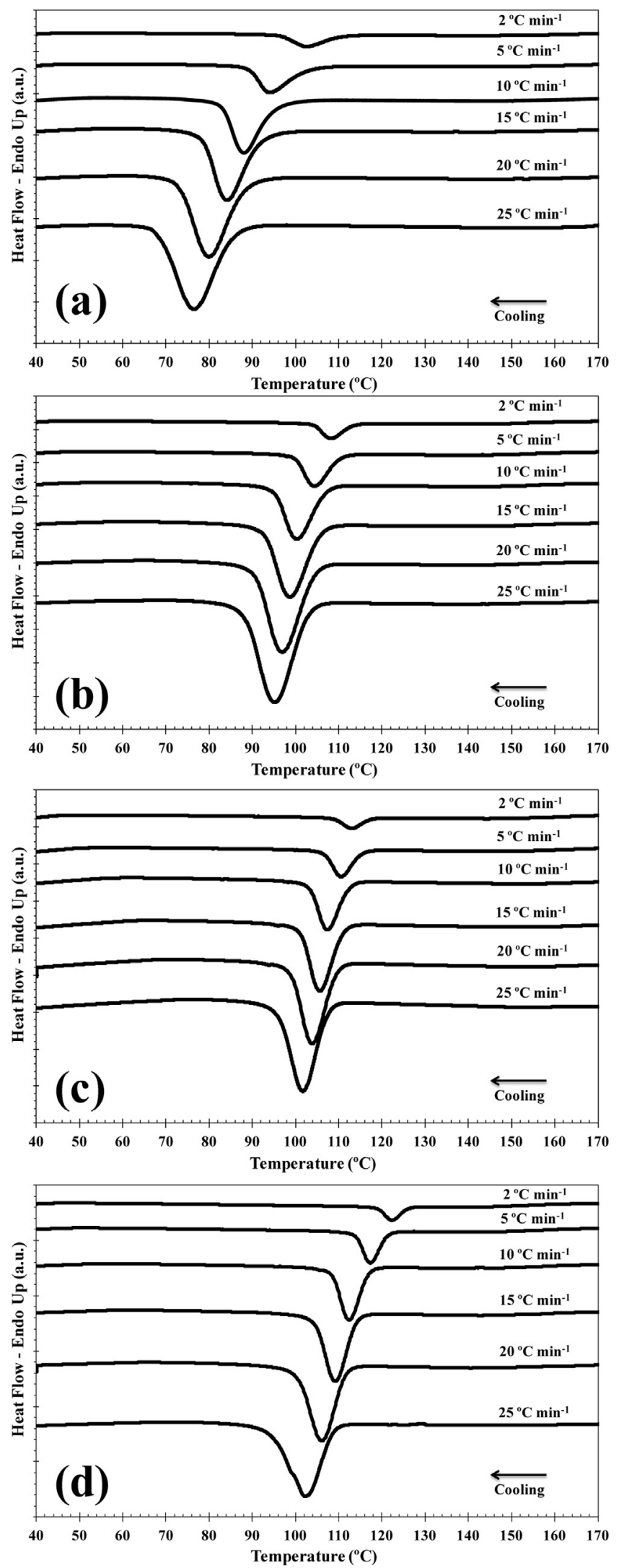

Fig. 3. DSC thermograms for non-isothermal crystallization of (a) PHBV and with PHBV/INT-WS 2 nanocomposites of (b) 0.1 , (c) 0.5 , and (d) 1.0 wt.\% of INT-WS 2 at indicated cooling rates. 
0.5 wt.\% $\quad 107.4{ }^{\circ} \mathrm{C}$, and $\mathrm{T}_{\mathrm{p}, 1.0 \mathrm{wt} . \%} \quad 112.4{ }^{\circ} \mathrm{C}$. Thus, the addition of INT $\mathrm{WS}_{2}$ had a remarkable heterogeneous nucleation capacity for accelerating the crystallization process of PHBV by providing stable nucleation platforms not observed in the control sample at higher temperatures. The data supports that both independent variables (cooling rates and INT $\mathrm{WS}_{2}$ concentrations) were responsible for the observed variations in $T_{p}$. This observation is quite reproducible for nanocomposites crystallized at different cooling rates (Table 1). Fig. 4 compares the $T_{p}$ values observed for the PHBV/INT WS 2 nanocomposites with those of PHB containing similar amounts of inorganic nanotubes. The data clearly shows that the addition of INT $\mathrm{WS}_{2}$ plays a remarkable role in accelerating the crystallization rate of PHBV. Nevertheless, for the same nanofiller loading, the improvements in $T_{p}$ for the PHBV based samples are lower than those observed for the corresponding binary PHB INTs composites [44]. This may attributed to the steric effect of ethyl side chain of HV units for the PHBV polymer crystallization.

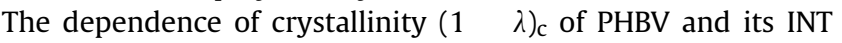
nanocomposites, shown in Table 1 both as a function of $\phi$ and INT concentration, almost mirrors the $T_{p}$ trends previously mentioned, and is expected because at slower $\phi$ the polymer chains have more time to organize into crystalline domains with fewer defects, and thus higher $\left(\begin{array}{ll}1 & \lambda\end{array}\right)_{\mathrm{c}}$. The addition of INT WS $\mathrm{W}_{2}$ showed a similar trend as a function of $\phi$ but a much higher $\left(\begin{array}{ll}1 & \lambda\end{array}\right)_{c}$ was observed as a result of earlier nucleation at higher temperatures where higher mobility facilitates chain entry into crystalline domains. Thus, very large increments in crystallinity were observed between the neat polymer and the nanocomposites, shown in Table 1 along with other dynamic crystallization data and results. In the same way, Fig. 5 compares the $\left(\begin{array}{ll}1 & \lambda\end{array}\right)_{c}$ values observed for the PHBV/INT WS nanocomposites with those of PHB containing similar amounts of inorganic nanotubes. In contrast to the results shown in Fig. 4, for

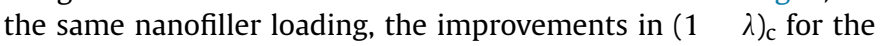
PHBV based samples are higher than those observed for the cor responding binary PHB INT composites [44].

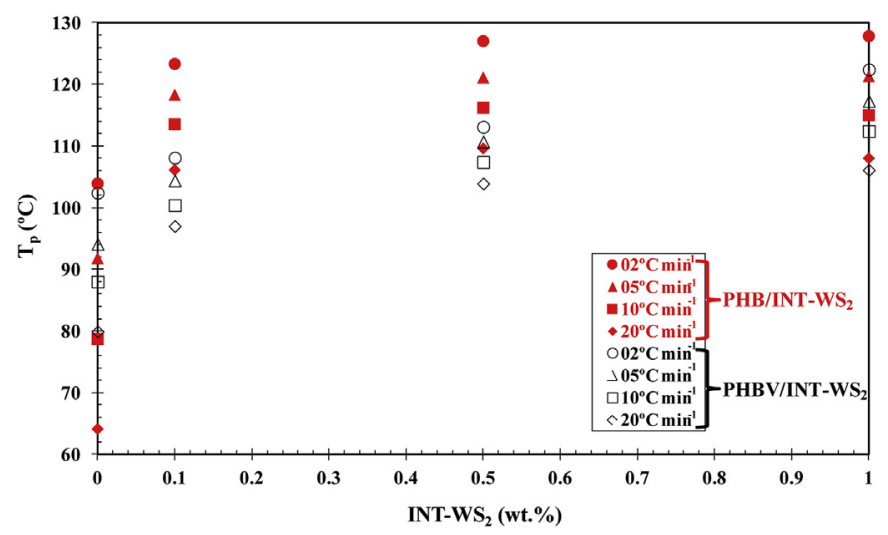

Fig. 4. Comparison of the crystallization temperature $\left(T_{p}\right)$ of PHBV and PHB [44] nanocomposites with INT-WS ${ }_{2}$ content at indicated cooling rates.

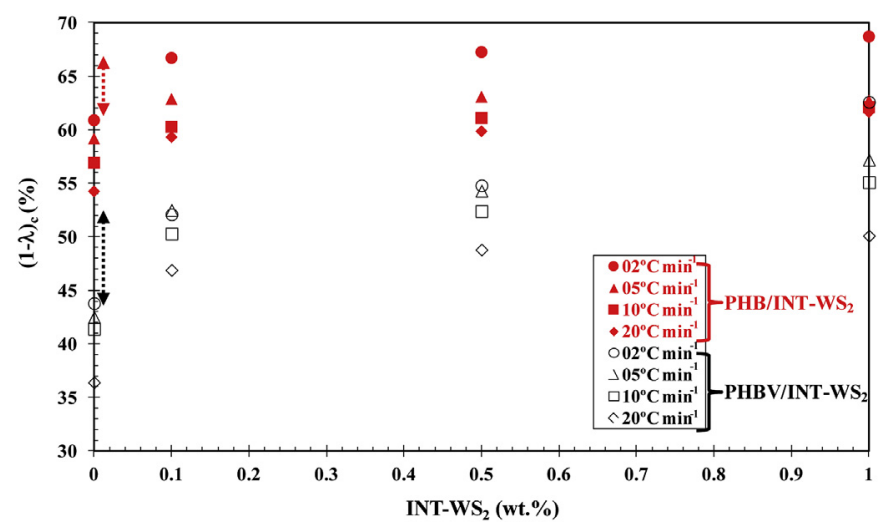

Fig. 5. Comparison of the crystallinity $(1-\lambda)_{\mathrm{c}}$ of PHBV and PHB [44] nanocomposites with INT-WS 2 content at indicated cooling rates.

Table 1

Non-isothermal crystallization parameters for PHBV/INT-WS ${ }_{2}$ nanocomposites.

\begin{tabular}{|c|c|c|c|c|c|}
\hline INT-WS 2 (wt.\%) & $\phi\left({ }^{\circ} \mathrm{C} \min { }^{1}\right)$ & $T_{p}\left({ }^{\circ} \mathrm{C}\right)$ & $(1-\lambda)_{c}(\%)$ & $\varphi^{\mathrm{a}}(0 \leq \varphi \leq 1)$ & $\Delta E^{\mathrm{b}}\left(\mathrm{kJ} \mathrm{mol}{ }^{1}\right)$ \\
\hline \multirow[t]{6}{*}{0.0} & 2 & 102.4 & 43.8 & 1.00 & -114.3 \\
\hline & 5 & 94.1 & 42.5 & & \\
\hline & 10 & 88.0 & 41.4 & & \\
\hline & 15 & 84.1 & 38.7 & & \\
\hline & 20 & 79.9 & 36.4 & & \\
\hline & 25 & 76.5 & 34.5 & & \\
\hline \multirow[t]{6}{*}{0.1} & 2 & 108.1 & 52.1 & 0.64 & -235.4 \\
\hline & 5 & 104.4 & 52.5 & & \\
\hline & 10 & 100.4 & 50.3 & & \\
\hline & 15 & 98.8 & 48.2 & & \\
\hline & 20 & 97.0 & 46.9 & & \\
\hline & 25 & 95.2 & 45.3 & & \\
\hline \multirow[t]{6}{*}{0.5} & 2 & 113.1 & 54.8 & 0.49 & -271.4 \\
\hline & 5 & 110.7 & 54.3 & & \\
\hline & 10 & 107.4 & 52.4 & & \\
\hline & 15 & 105.7 & 50.9 & & \\
\hline & 20 & 103.9 & 48.8 & & \\
\hline & 25 & 101.7 & 46.4 & & \\
\hline \multirow[t]{6}{*}{1.0} & 2 & 122.4 & 62.6 & 0.44 & -163.2 \\
\hline & 5 & 117.3 & 57.2 & & \\
\hline & 10 & 112.4 & 55.1 & & \\
\hline & 15 & 109.2 & 52.4 & & \\
\hline & 20 & 106.1 & 50.1 & & \\
\hline & 25 & 102.3 & 47.8 & & \\
\hline
\end{tabular}

\footnotetext{
a Nucleation activity $(\varphi)$ calculated with Dobreva Gutzow equation.
}

b Effective energy barrier $(\Delta \mathrm{E})$ calculated with Kissinger equation. 


\subsection{Nucleation activity}

Dobreva and Gutzow [47,48] suggested a simple method for calculating the nucleation activity of foreign substrates in polymer melts. Nucleation activity $(\varphi)$ is a factor by which the work of three dimensional nucleation decreases with the addition of a foreign substrate. If the foreign substrate is extremely active, $\varphi$ approaches 0 , while for inert particles, $\varphi$ approaches 1 . The nucleation activity $(\varphi)$ of the INT $\mathrm{WS}_{2}$ at various nanofiller concentrations was deter mined by the ratio:

$\varphi \quad \frac{B^{*}}{B}$

where $B$ is a parameter for the pristine polymer, and $B^{*}$ is for the polymer/nucleator system. $B$ and $B^{*}$ can both be experimentally determined from the slope of the following equation:

$\ln \phi \quad A \quad \frac{B\left(o r B^{*}\right)}{\Delta T_{p}^{2}}$

where $\phi$ is the cooling rate, $A$ is a constant, $\Delta T_{p}$ is the supercooling $\left(\begin{array}{ll}T_{m} & T_{p}\end{array}\right), T_{m}$ is the melting temperature (data not shown) and $T_{p}$ is the crystallization peak temperature. A linear relationship was obtained for each sample, Fig. 6. The values of $B$ and $B^{*}$ are obtained from the slope of the fitted lines, and the $\varphi$ calculated from this ratio. Values of $\varphi \quad 0.64,0.48$, and 0.44 were obtained for the 0.1 , 0.5 , and 1.0 wt.\% nanocomposites, respectively (Table 1 ). The nanocomposite with the lowest concentration ( $0.1 \mathrm{wt} . \%)$ presented a much lower value of $\varphi$ than that of pure PHBV $\left(\begin{array}{ll}\varphi & 1\end{array}\right)$, showing that INT WS $\mathrm{W}_{2}$ effectively contribute to the nucleation of PHBV, and higher concentrations showed even lower values of $\varphi$ although tended to asymptote. These results demonstrate the excellent nucleating effect of INT $\mathrm{WS}_{2}$ on the crystallization of PHBV. In order to put this into perspective the nucleating capacity of INT $\mathrm{WS}_{2}$ was compared with that of other PHBV based composite materials re ported in the literature incorporating nucleating agents (NA) for PHBV, such as carbon nanotubes, halloysite nanotubes, etc. where the nanosized fillers were shown to have the added advantage of acting as reinforcements for the polymer. Since the intrinsic properties of the different PHBV copolyesters employed in the re ported studies (such as molecular weight and HV content) varied, the increment of crystallization peak temperature, $\Delta T$ between that of the neat polymer and the composite materials, defined as

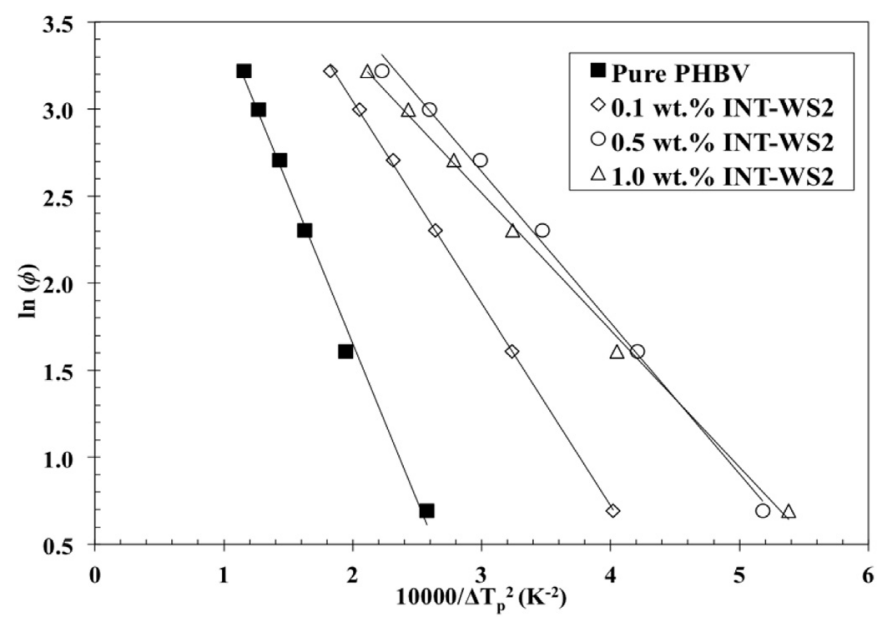

Fig. 6. Dobreva Gutzow plots for PHBV/INT-WS 2 nanocomposites.

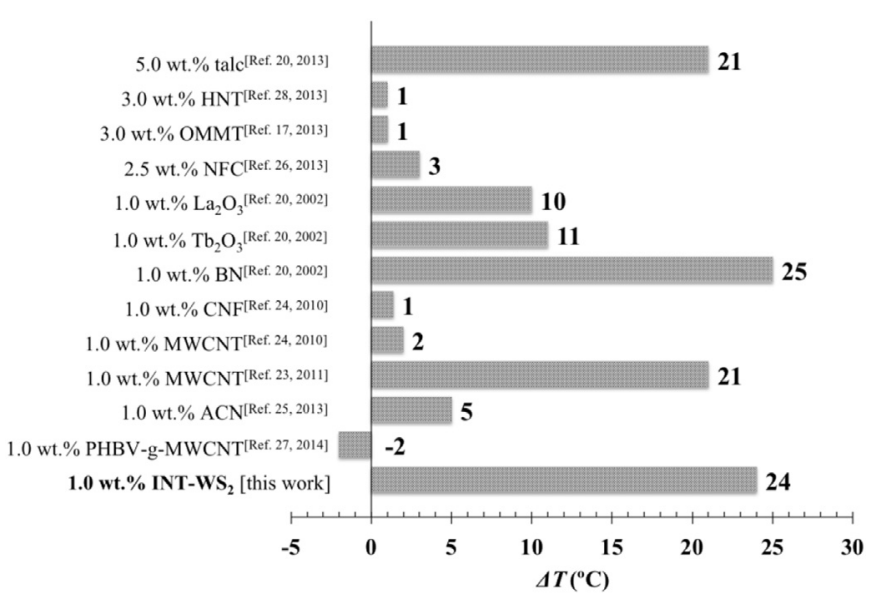

Fig. 7. Increment of $T_{p}$ for various fillers studied to improve the crystallization of PHBV.

$\Delta T T_{p, P H B V+N A} T_{p, P H B V}$, was calculated, allowing a direct com parison. In all cases, $\Delta T$ was at a common rate of $\phi \quad 10^{\circ} \mathrm{C} \mathrm{min}^{-1}$.

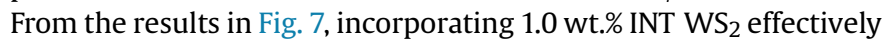
increased the peak crystallization temperature to a value compa rable to that of $\mathrm{BN}$, and greater than all other NAs. BN is currently estimated to be more expensive than INT $\mathrm{WS}_{2}$ and, like talc, is not a nanoparticle filler, also possibly leading to deficient mechanical reinforcement qualities that are observed with other nano sized filler materials [20]. Also noteworthy, MWCNT filled PHBHV [23] showed an appreciable $\Delta T$ but the nanotubes were chemically modified to incorporate carboxyl group tails on the nanotube sur face to reduce surface tension for better dispersion within the PHBV matrix. Non modified MWCNT [24] showed a low $\Delta T$ attributed due to poorer dispersion, as mentioned before. Whilst the available literature on NA for PHBV is relatively limited, the data presented here shows that INT $\mathrm{WS}_{2}$ is one of the best alternatives currently available.

\subsection{Effective energy barrier}

Another common correlation used in the characterization of the non isothermal crystallization behavior is the effective (or activa tion) energy barrier $(\Delta \mathrm{E})$. This describes macromolecular chain mobility and crystal growth ability of a polymer during the crys tallization process from the Kissinger equation [49] using crystal lization peak temperatures $T_{p}$ for given cooling rates, $\phi$ :

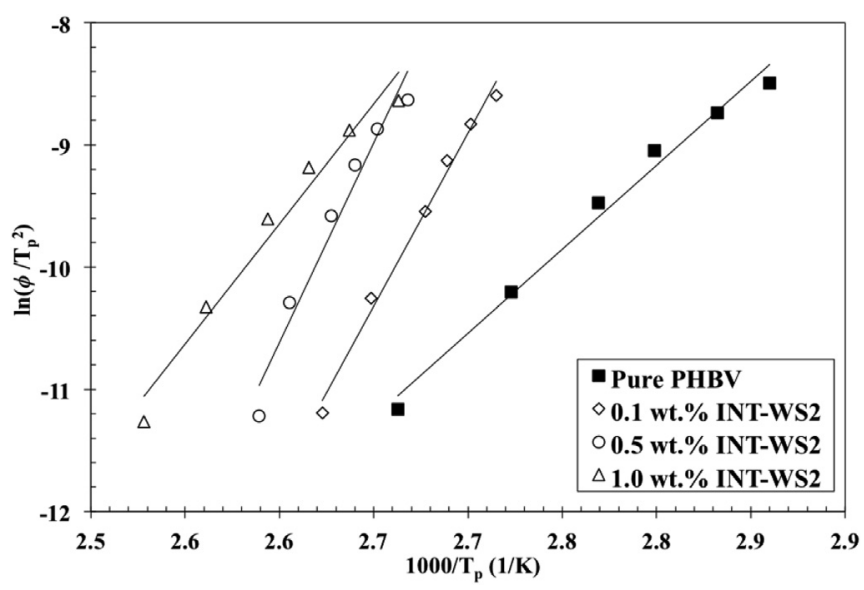

Fig. 8. Kissinger plots for PHBV/INT-WS $\mathrm{W}_{2}$ nanocomposites. 
$\ln \left(\frac{\phi}{T_{p}^{2}}\right) \quad$ Constant $\frac{\Delta E}{R T_{p}}$

where $\Delta E$ is the effective energy barrier and $R$ is the universal gas constant. The activation energies for the four samples were calcu lated by plotting $\ln \left(\phi / T_{p}^{2}\right)$ against $1 / T_{p}$ to generate the linear slopes of $\Delta E / R$ shown in Fig. 8 (data in Table 1 ). The resulting slopes indicated that all nanocomposites presented lower energies than that of the neat sample. Thus, $\Delta E$ of the neat polymer is about $114.3 \mathrm{~kJ} \mathrm{~mol}^{-1}$ and decreases by $106 \%, 137 \%$, and $43 \%$ upon addition of $0.1,0.5$ and 1.0 wt.\% INT $\mathrm{WS}_{2}$, respectively (i.e.
$\Delta \mathrm{E}_{0.1}$

$\Delta \mathrm{E}_{1.0}$ wt.\%

$235.4 \mathrm{~kJ} \mathrm{~mol}^{-1}, \Delta \mathrm{E}_{0.5} \mathrm{wt} . \%$

$271.4 \mathrm{~kJ} \mathrm{~mol}^{-1}$ and molecular chain transport on the growing crystal surfaces. The in crease in the apparent $\Delta E$ in the $1.0 \mathrm{wt} . \%$ nanocomposite with respect to the lower concentration samples indicates that any re striction of molecular mobility of PHBV chains through volume does not appear to be a limiting factor on the crystallization rate, and since the $T_{p}$ for this composition appears at higher tempera tures, it further demonstrates that the nucleation activity of these inorganic nanotubes plays a dominant role in accelerating the crystallization process of PHBV. The reduction of the $\Delta E$ obtained in this work are lower than those observed for the corresponding binary PHB INT composites $\left(\Delta \mathrm{E}_{\mathrm{PHB}} \quad 76 \mathrm{~kJ} \mathrm{~mol}^{-1}\right.$, (a)

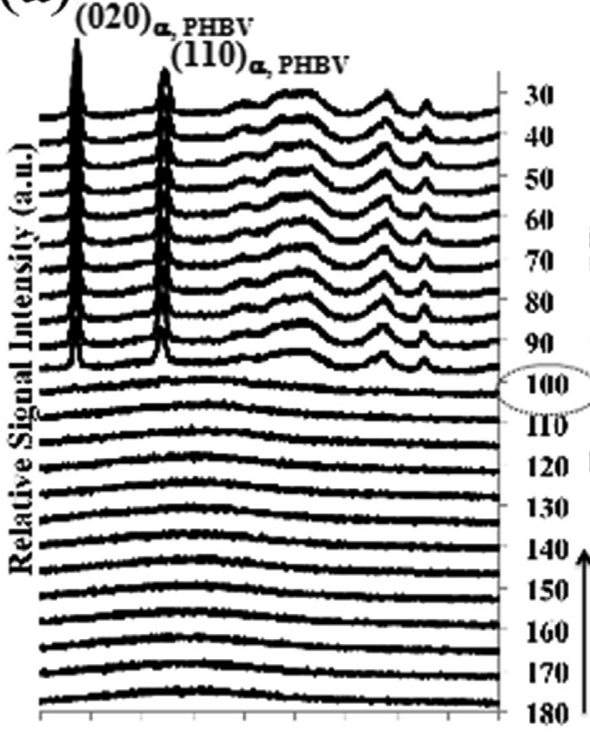

$\begin{array}{llllllllll}12 & 14 & 16 & 18 & 20 & 22 & 24 & 26 & 28 & 30\end{array}$

$20\left({ }^{\circ}\right)$

(c)

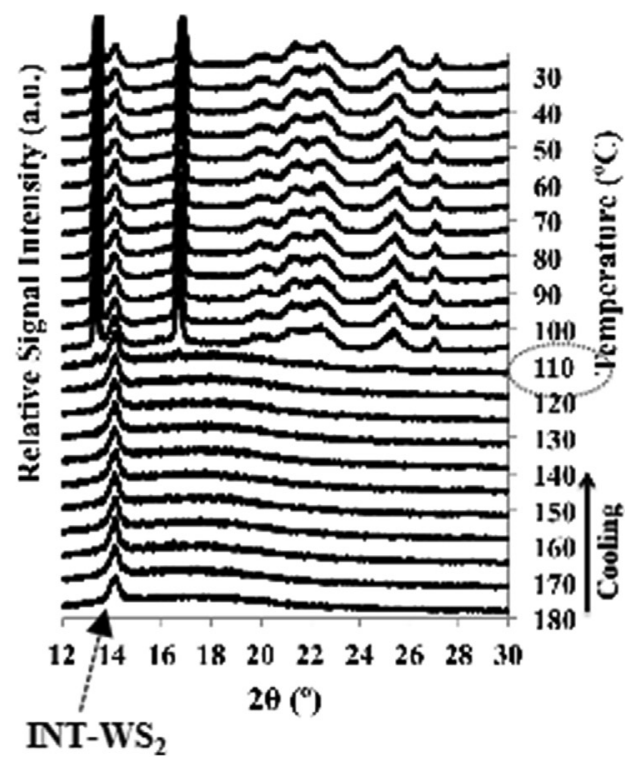

(b)

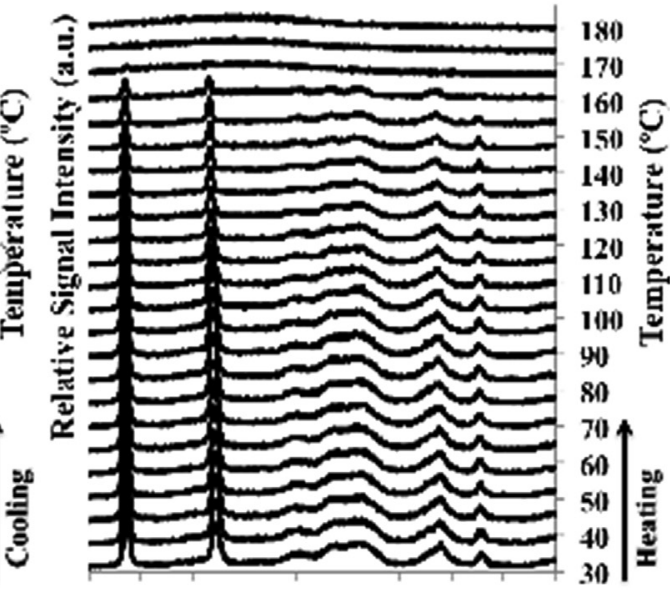

$\begin{array}{llllllllll}12 & 14 & 16 & 18 & 20 & 22 & 24 & 26 & 28 & 30\end{array}$

$2 \theta(\%)$

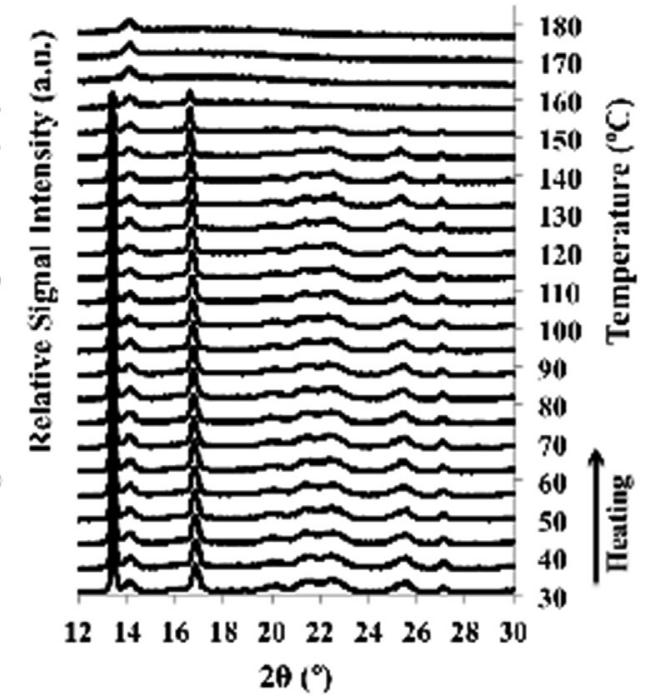

Fig. 9. WAXS diffractograms of neat PHBV and PHBV/INT-WS 2 (1.0 wt.\%) obtained during cooling from the melt to room temperature and subsequent heating. 
$\Delta \mathrm{E}_{0.1}$ wt.\%

$232 \mathrm{~kJ} \mathrm{~mol}^{-1}, \Delta \mathrm{E}_{0.5}$ wt.\%

$\Delta \mathrm{E}_{1.0}$ wt.\% $200 \mathrm{~kJ} \mathrm{~mol}^{-1}$ ) [44].

\subsection{Crystal structure and morphology}

Wide angle $X$ ray diffraction (WAXS) measurements were made to determine whether the addition of INT $\mathrm{WS}_{2}$ affects the crystal form of the PHBV, and the evolution of the crystal structure during crystallization and melting was investigated in a temperature controlled chamber, with cooling and heating rates of approxi mately $7^{\circ} \mathrm{C} \mathrm{min}^{-1}$, close to the values of $\phi$ in the DSC experiments. Fig. 9 presents representative WAXS diffractograms obtained dur ing crystallization and subsequent melting for neat PHBV and a nanocomposite with 1.0 wt.\% INT WS 2 . For both the neat PHBV and the PHBV/INT WS 2 (1.0 wt.\%) samples it can be seen that the crystalline structure developed was identical, with diffraction peaks at $2 \theta \quad 13.4^{\circ}, 16.8^{\circ}, 20.1^{\circ}, 21.5^{\circ}, 22.5^{\circ}, 25.5^{\circ}, 27.2^{\circ}$, the first two being the most intense corresponding to planes (020) and (110), in respective order, characteristic of orthorhombic PHBV crystalline $\alpha$ type structure $[8,50]$. The nanocomposite sample presented an additional diffraction peak at $14.2^{\circ}$, which corre sponds to the (002) plane of the inorganic nanotubes [30]. On the other hand, the pure PHBV crystallized at around $90-100{ }^{\circ} \mathrm{C}$, in close agreement with the DSC results (i.e. at $\phi \quad 5{ }^{\circ} \mathrm{C} \min ^{-1}, T_{p}$, PHBV $\quad 94.1^{\circ} \mathrm{C}$ ). Similarly, the WAXS data also confirms crystalli zation onset at higher temperatures for the $1.0 \mathrm{wt}$ \% sample compared to neat PHBV.

In addition to the above WAXS observation, polarized optical microscopy (POM) was used to visually examine the crystalline morphology of PHBV and the nanocomposites, in particular their
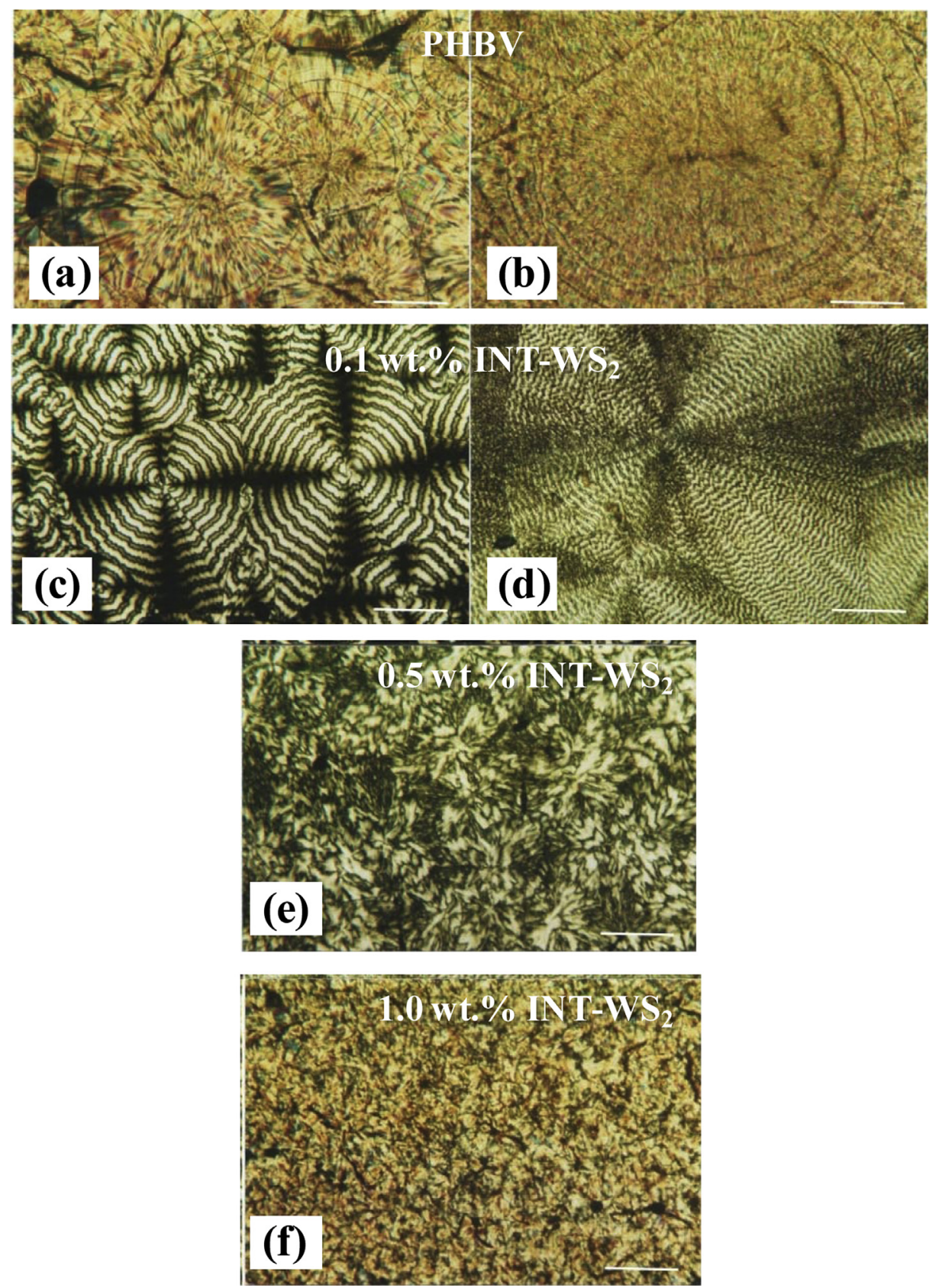

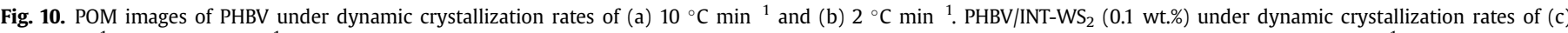

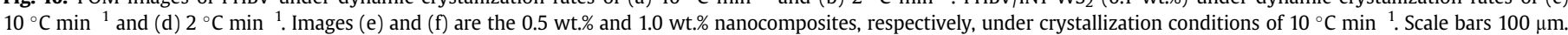


spherulitic structure. Under dynamic crystallization conditions, neat PHBV and (particularly) the $0.1 \mathrm{wt} . \%$ samples showed ring banded spherulites with concentric bands and Maltese crosses af ter cooling to room temperature at 10 and $2{ }^{\circ} \mathrm{C} \mathrm{min}-1$ as shown in Fig. 10. The observed ring bands are thought to originate from either stress induced twisting [51-53] or other non lamellar twisting phenomena [54]. However, the increase of concentration of INT $\mathrm{WS}_{2}$ (0.5 and $1.0 \mathrm{wt}$.\%) clearly disturbed spherulite forma tion, possibly due to the dramatic increase of the nucleation density and faster crystal formation of PHBV, which were consistent with the DSC results in the previous section.

\section{Conclusions}

In the present work, a series of biopolymer PHBV/INT $\mathrm{WS}_{2}$ nanocomposites have been successfully prepared at various INT $\mathrm{WS}_{2}$ loadings ranging from 0.1 to $1.0 \mathrm{wt}$.\%, without the use of sur face modifiers. The non isothermal crystallization behavior and kinetics were studied, and the results demonstrated that the INT $W_{2}$ content are very efficient factors for controlling overall crys tallization rate and crystallinity of PHBV. Compared to those of neat PHBV, the crystallization rate and the nucleation density of PHBV matrix of the composites were improved remarkably due to a strong heterogeneous nucleating effect of INT $\mathrm{WS}_{2}$ in the matrix. Nevertheless, for the same nanofiller loading, the improvements in $T_{p}$ for the PHBV based samples are lower than those observed for the corresponding binary PHB INT nanocomposites. This may attributed to the steric effect of ethyl side chain of $\mathrm{HV}$ units for the PHBV polymer crystallization. The well dispersed INT $\mathrm{WS}_{2}$ nano particles in the nanocomposites enhanced the nucleation capacity and crystallinity of PHBV matrix without altering its crystal struc ture. The nucleation activity of INT $\mathrm{WS}_{2}$ plays a crucial role in accelerating the crystallization rate of PHBV, reducing the effective barrier energy compared to neat PHBV. In particular, it was shown that INT $\mathrm{WS}_{2}$ exhibits a much more prominent nucleation activity on the crystallization of PHBV than other possible nucleating agents or nano sized fillers. More importantly, these nanocomposites also show strong improvement in the thermal stability in comparison to those of PHB containing similar amounts of inorganic nanotubes. These features may be advantageous for the enhancement of the mechanical properties and processability of PHBV based materials. The good thermal properties and desired morphology obtained by incorporating INT $\mathrm{WS}_{2}$ in PHBV can enhance its use as a low cost renewable and environmentally friendly biopolymer material for many applications.

\section{Acknowledgments}

Financial support for this work was provided by the Spanish Ministry Economy and Competitivity (MINECO) (Projects MAT 201021070 C02 01 and MAT2013 41021 P). MN would also like to acknowledge the MINECO for a 'Ramón y Cajal' Senior Research Fellowship. Very special thanks and appreciation go to Dr. Alla Zak for providing the $\mathrm{WS}_{2}$ inorganic nanotubes that made this research possible.

\section{References}

[1] M.M. Reddy, S. Vivekanandhan, M. Misra, S.K. Bhatia, A.K. Mohanty, Prog Polym. Sci. 38 (2013) 16531689.

[2] B. Laycock, P. Halley, S. Pratt, A. Werker, P. Lant, Prog. Polym. Sci. 39 (2014) 397442.

[3] S. Povolo, M.G. Romanelli, M. Basaglia, V.I. Ilieva, A. Corti, A. Morelli,
E. Chiellini, S. Casella, New Biotechnol. 30 (2013) 629634.

[4] B. Laycock, M.V. Arcos-Hernandez, A. Langford, S. Pratt, A. Werker, P.J. Halley, P.A. Lant, New Biotechnol. 31 (2014) 345356.

[5] K. Sudesh, H. Abe, Y. Doi, Prog. Polym. Sci. 25 (2000) 15031555.

[6] A.A. Shah, F. Hasan, A. Hameed, S. Ahmed, Biotechnol. Adv. 26 (2008) 246265.

[7] I. Noda, P.R. Green, M.M. Satkowski, L.A. Schechtman, Biomacromolecules 6 (2005) 580586.

[8] T.L. Bluhm, G.K. Hamer, R.H. Marchessault, C.A. Fyfe, R.P. Veregin, Macromolecules 19 (1986) 28712876.

[9] N. Hosoda, E.H. Lee, T. Tsujimoto, H. Uyama, Ind. Eng. Chem. Res. 52 (2013) 15481553.

[10] S. Modi, K. Koelling, Y. Vodovotz, Eur. Polym. J. 49 (2013) 36813690.

[11] A. Buzarovska, A. Grozdanov, J. Mater. Sci. 44 (2009) 18441850.

[12] A. Bianco, M. Calderone, I. Cacciotti, Mater. Sci. Eng. C 33 (2013) 10671077.

[13] L. Miao, Z. Qiu, W. Yang, T. Ikehara, React. Funct. Polym. 68 (2008) 446457.

[14] R.P. Pearce, R.H. Marchessault, Macromolecules 27 (1994) 38693874.

[15] A. Javadi, Y. Srithep, J. Lee, S. Pilla, C. Clemons, S. Gong, L.S. Turng, Compos. A 46 (2010) 982990.

[16] J. Tao, C. Song, M. Cao, D. Hu, L. Liu, N. Liu, S. Wang, Polym. Degrad. Stab. 94 (2009) 575583.

[17] M.C. Branciforti, M.C. Silveira Corrêa, E. Pollet, J.A. Marcondes Agnelli, P.A. Nascente, L. Avérous, Polym. Test. 32 (2013) 12531260.

[18] A. Buzarovska, G. Bogoeva-Gaceva, A. Grodzdanov, M. Avella, G. Gentile, M. Errico, J. Mater. Sci. 42 (2007) 65016509.

[19] S. Singh, A.K. Mohanty, M. Misra, Compos. A 41 (2010) 304312.

[20] W.J. Liu, H.L. Yang, Z. Wang, L.S. Dong, J.J. Liu, J. Appl. Polym. Sci. 86 (2002) 21452152.

[21] P. Pan, G. Shan, Y. Bao, Z. Weng, J. Appl. Polym. Sci. 129 (2013) 13741382.

[22] A. Javadi, Y. Srithep, S. Pilla, J. Lee, S. Gong, L.S. Turng, Mater. Sci. Eng. C 30 (2010) 749757

[23] G.F. Shan, X. Gong, W.P. Chen, L. Chen, M.F. Zhu, Colloid Polym. Sci. 289 (2011) 10051014.

[24] M.D. Sanchez-Garcia, J.M. Lagaron, S.V. Hoa, Compos. Sci. Technol. 70 (2010) 10951105.

[25] B. Wang, J. Li, J. Zhang, H. Li, P. Chen, Q. Gu, Z. Wang, Carbohydr. Polym. 95 (2013) 100106

[26] Y. Srithep, T. Ellingham, J. Peng, R. Sabo, C. Clemons, L.S. Turng, S. Pilla, Polym. Degrad. Stab. 98 (2013) 14391449.

[27] H.Y. Yu, Z.Y. Qin, B. Sun, X.G. Yang, J.M. Yao, Compos. Sci. Technol. 94 (2014) 96104.

[28] L.N. Carli, T.S. Daitx, G.V. Soares, J.S. Crespo, R.S. Mauler, Appl. Clay Sci. 87 (2014) 311319.

[29] R. Tenne, G. Seifert, Annu. Rev. Mater. Res. 39 (2009) 387413.

[30] M. Krause, A. Mücklich, A. Zak, G. Seifert, S. Gemming, Phys. Status Solidi B 248 (2011) 27162719.

[31] R. Tenne, M. Redlich, Chem. Soc. Rev. 39 (2010) 14231434.

[32] A. Zak, L. Sallacan-Ecker, A. Margolin, M. Genut, R. Tenne, Nano 4 (2009) 9198.

[33] M. Remškar, Inorganic nanotubes, Adv. Mater. 16 (2004) 14971504.

[34] A. Zak, L. Sallacan-Ecker, R. Efrati, L. Drangai, N. Fleischer, R. Tenne, Sens. Transducers J. 12 (2011) 110.

[35] Y.Q. Zhu, W.K. Hsu, N. Grobert, B.H. Chang, M. Terrones, H. Terrones, H.W. Kroto, D.R.M. Walton, Chem. Mater. 12 (2000) 11901194.

[36] A. Zak, L. Sallacan-Ecker, A. Margolin, Y. Feldman, R. Popovitz-Biro, A. AlbuYaron, M. Genut, R. Tenne, Fuller. Nanotub. Carbon Nanostruct. 19 (2010) 1826.

[37] A.R. Adini, M. Redlich, R. Tenne, J. Mater. Chem. 21 (2011) 1512115131.

[38] R. Tenne, R. Rosentsveig, A. Zak, Phys. Status Solidi A 210 (2013) 22532258.

[39] M. Pardo, T. Shuster-Meiseles, S. Levin-Zaidman, A. Rudich, Y. Rudich, Environ. Sci. Technol. 48 (2014) 34573466.

[40] M. Naffakh, M. Remškar, C. Marco, M.A. Gómez, I. Jiménez, J. Mater. Chem. 21 (2011) 35743578.

[41] E. Zohar, S. Baruch, M. Schneider, H. Dodiuk, S. Kenig, D.H. Wagner, A. Zak, R. Tenne, J. Adhes. Sci. Technol. 25 (2011) 16031617.

[42] C.S. Reddy, A. Zak, E. Zussman, J. Mater. Chem. 21 (2011) 1608616093.

[43] M. Naffakh, C. Marco, G. Ellis, CrystEngComm 16 (2014) 50625072.

[44] M. Naffakh, C. Marco, G. Ellis, CrystEngComm 16 (2014) 11261135.

[45] M. Naffakh, C. Marco, G. Ellis, S.R. Cohen, A. Laikhtman, L. Rapoport, A. Zak, Mater. Chem. Phys. 147 (2014) 273284.

[46] M. Naffakh, A.M. Diez-Pascual, J. Mater. Chem. B 2 (2014) 45094520.

[47] A. Dobreva, I. Gutzow, J. Non Cryst. Solids 162 (1993) 112.

[48] A. Dobreva, I. Gutzow, J. Non Cryst. Solids 162 (1993) 1325.

[49] H.E. Kissinger, J. Res. Nat. Bur. Stand. 57 (1956) 217221.

[50] A.J. Owen, J. Heinzel, Ž. Škrić, V. Divjakoić, Polymer 33 (1992) 15631567.

[51] A. Keller, J. Polym. Sci. 17 (1955) 291308.

[52] A. Keller, J. Polym. Sci. 17 (1955) 351364

[53] A. Keller, J.R.S. Waring, J. Polym. Sci. 17 (1955) 447472.

[54] A. Meyer, K.C. Cheng, S.H. Li, S. Forster, E.M. Woo, Ind. Eng. Chem. Res. 49 (2010) 1208412092. 